\title{
Games of Social Interactions with Local and Global Externalities*
}

\author{
Michel Le Breton ${ }^{\dagger} \quad$ Shlomo Weber ${ }^{\ddagger}$
}

September 2009

\begin{abstract}
In this short paper we introduce a general class of games that allow for local (dyadic) and global types of social interactions. By constructing a "potential", we show that every game in this class admits a Nash equilibrium in pure strategies. We illustrate the universality of the result through a large spectrum of applications in various disciplines.
\end{abstract}

Keywords: Social interactions, conformity, local externalities, potential function, Nash equilibrium.

JEL Numbers: C72, D74, D85.

\section{Introduction}

Suppose, we need to decide which laptop to purchase and assume, for simplicity, that all options are reduced to two choices, PC and MAC. In trying to make a decision, we obviously rely on our intrinsic preferences and a potential benefit based on laptops' features, design, price and our prior computer experience. However, the process does not stop there and we often invoke elements of "social interaction" that can manifest itself in two ways. One is local, when we consult, often on bilateral basis, with our

* The paper has been presented at the seminars in New Economic School, the Central Economics and Mathematics Institute, Moscow, Universities of Malaga and Seville, SMU, and the conference "Rationality, behavior and experiments" at the Higher School of Economics, Moscow. We wish to thank their participants and, especially, Nikolai Kukushkin, Thomas Palfrey and Jörgen Weibull for useful comments.

$\dagger^{\dagger}$ Toulouse School of Economics, GREMAQ and IDEI, Toulouse, France.

${ }^{\ddagger}$ Department of Economics, Southern Methodist University, USA. 
peers (colleagues, co-authors, friends, family members, neighbors). Another is global, which represents the influence exerted by a global "market appeal", that tends to tilt our choice towards more popular product.

The dependence of individuals' utility on the actions and perception of others has been recognized in various studies of social interactions and social networks. ${ }^{1}$ The purpose of this note is to examine environments that exhibit both local and global influences. More specifically, we consider a class of normal form games where player's payoff for any given action is determined by her intrinsic preference for actions (taste component); dyadic externalities exerted by her peers on bilateral basis (local social interaction component $)^{2}$; the number of other players choosing this player's action (global social interaction component $)^{3}$.

We show that, in presence of symmetric dyadic externalities, where each pair of players influences each other in the same way, and a positive global effect, some mild continuity assumptions guarantee that our game admits a pure strategies Nash equilibrium. To prove this result, we demonstrate that our game has a potential (Rosenthal (1973), Monderer and Shapley (1996)), whose maximum over the set of all strategy profiles yields a Nash equilibrium of our game. ${ }^{4}$ We show moreover that if the set of feasible actions for each player is finite, the symmetry of dyadic influences is sufficient to ensure the existence of a pure strategies Nash equilibrium.

In the next section we introduce the model and state our result. Sections 3 and 4 contain various applications of our model.

\footnotetext{
${ }^{1}$ See, e.g., Becker (1974), Schelling (1978), Akerlof (1997), Glaeser, Sacerdote and Scheinkman (1996), Blume (1993), Durlauf (1999,2003), Manski (2000), Blume and Durlauf (2001, 2003), Brock and Durlauf (2001, 2002), Brock and Durlauf (2002), Glaeser and Scheinkman (2003), Galeotti at al. (2006), Jackson (2008)), among many others.

${ }^{2}$ Dyadic influence matrices have been utilized in empirical studies of various international conflicts. See, e,g., Bueno de Mesquita (1975), Axelrod and Bennett (1993), Fafchamps and Gubert (2007).

${ }^{3}$ The global component of social interaction is often called a conformity effect, driven by prestige, esteem, popularity or social acceptance (Akerlof (1980), Jones (1984), Bernheim (1994).

${ }^{4}$ The problem of maximizing the potential is quite challenging, however. It is NP-hard even in the case of two feasible actions for each player AND in absence of two out of the three components of the payoff functions above. In fact, this problem is equivalent to the celebrated MAX-CUT problem in combinatorial optimization.
} 


\section{The Model and Results}

Consider a class of games $G$ with a finite set of players (society) $N=\{1,2, \ldots, n\}$. Each player $i$ has a set of feasible actions (pure strategies) $X_{i}$. Let $X=\bigcup_{i=1}^{n} X_{i}$. For simplicity, we assume that all $X_{i}$ (and, thus, $X$ ) are compact subsets of the Euclidean space $\Re^{T}, T \geq 1$. Players' choices $x_{i} \in X_{i}$ generate the $n$-dimensional strategies profile $\mathbf{x}=\left(x_{1}, x_{2}, \ldots, x_{n}\right)$, which yields the partition of $N$ into clusters of players choosing the same strategies. We denote this partition by $\pi(\mathbf{x})$ and let $S^{i}(\mathbf{x})$ be the cluster in $\pi(\mathbf{x})$ which contains player $i$, as well as all other players $j$ for whom $x_{j}=x_{i}$. The payoff $U_{i}(\mathbf{x})$ of player $i$ is the sum of three terms:

$$
U_{i}(\mathbf{x})=V_{i}\left(x_{i}\right)+\sum_{j \in N} W_{i}^{j}\left(x_{i}, x_{j}\right)+H\left(x_{i},\left|S^{i}(\mathbf{x})\right|\right),
$$

where $|Z|$ stands for the cardinality of the set $Z$. The first term describes the intrinsic taste of player $i$ for her chosen action $x_{i}$. The second term represents the local dyadic social influences of others on player $i,{ }^{5}$ whereas the last term captures a conformity facet of social influence represented by the number of players who have chosen the same action. We impose the following assumptions:

Assumption A.1: Upper Semi-continuity. The following functions are upper semicontinuous: $V_{i}(\cdot): X^{i} \rightarrow \Re$ for all $i \in N, W_{i}^{j}(\cdot, \cdot): X^{i} \times X^{j} \rightarrow \Re$ for all $i, j \in N$ and $H(\cdot, r): X \rightarrow \Re$ for all $r, 1 \leq r \leq n$.

Assumption A.2: Symmetry. $W_{i}^{j}\left(x_{i}, x_{j}\right)=W_{j}^{i}\left(x_{j}, x_{i}\right)$ for every $i, j \in N$, every $x_{i} \in X_{i}$ and every $x_{j} \in X_{j}$. Let also $W_{i}^{i}(x, x)=0$ for every $i \in N$ and every $x \in X_{i}$.

Assumption A.3: Conformity. $H(x, \cdot)$ is increasing for all $x \in X$.

Assumption A.1 is of a technical nature. Assumption A.2 implies the symmetry of dyadic influence between any two players $i$ and $j$. Assumption A.3 represents the conformity effect. Our main result is:

Theorem: Under A1, A2, A3, every game in $G$ admits a Nash equilibrium in pure strategies.

\footnotetext{
${ }^{5}$ We may explicitly introduce a notion of "peer groups" by simply assuming that the values $W_{i}^{j}$ are equal to zero if $i$ and $j$ do not belong to same peer group.
} 
The proof of the theorem, which is relegated to the Appendix, utilizes the ingenious idea of Rosenthal (1973) to show that our game of social interactions is a potential game studied in Monderer and Shapley (1996) (see also Kukushkin (2007)).

Note that Assumption A1 is vacuous when $X$ is a finite set. Also in that case, we can dispense with the conformity assumption A3, and, moreover, allow for "congestion effects" when individuals' utility is adversely affected by the number of others making the same choice (Milchtaich (1996), Konishi, Le Breton, Weber (1997b)).

Corollary: If the set $X$ is finite, then under A2, every game in $G$ admits a Nash equilibrium in pure strategies.

\section{Linear-Quadratic Games}

We now turn to the examination of environments covered by our result and begin with a class of social interaction games where the taste and dyadic components in $(*)$ consist of linear and quadratic terms.

\subsection{Network Games}

Akerlof (1997) considered a status model where strategic choices of all players represent a unidimensional interval and a individual utility depends on the difference between her own status and the status of others within the society:

$$
U_{i}(\mathbf{x})=-a x_{i}^{2}+b x_{i}-d \sum_{j \neq i}\left(x_{j}-x_{i}\right)
$$

where $a, b, c, d$ are positive constants and $x$ is an effort, or a status-producing variable. Akerlof (1997) also considered a conformity model, ${ }^{6}$ where individuals minimize the social distance from their peers:

$$
U_{i}(\mathbf{x})=-a x_{i}^{2}+b x_{i}+c-d \sum_{j \neq i}\left|x_{j}-x_{i}\right| .
$$

Ballester, Calvó-Armengol and Zenou (2006) studied the contribution game with the following specification of utilities:

$$
U(\mathbf{x})=-a x_{i}^{2}+b x_{i}^{2}+\sum_{j \neq i} \sigma_{i j} x_{i} x_{j}
$$

\footnotetext{
${ }^{6}$ See Bernheim (1994) for a more general functional form.
} 
where, $a, b>0, x_{i}$ is $i$ 's contribution and the dyadic social influences are captured by the cross-derivatives $\frac{\partial^{2} U_{i}}{\partial x_{i} \partial x_{j}}=\sigma_{i j}=\sigma_{j i}, i \neq j$. When $\sigma_{i j}>0$, the contributions by $i$ and $j$ are strategic complements, whereas when $\sigma_{i j}<0$, these two efforts are strategic substitutes. A variant of this model has been examined by Corbo, CalvóArmengol and Parkes (2007), where $U(\mathbf{x})=-\frac{x_{i}^{2}}{2}+x_{i}+\theta \sum_{j \neq i} \sigma_{i j} x_{i} x_{j}$, where $\theta$ can be either negative or positive and $\sigma_{i j}=\sigma_{i j} \in\{0,1\}$ for all $i \neq j$. Again, the case where $\theta<0$ can be considered as a variant of the contribution game with free ride incentives. A particular case of this specification is considered by Glaeser, Sacerdote and Scheinkman (2003) in their exploration of a social multiplier, where the value $\sigma_{i j}$ is equal to $1 /\left(\left|P_{m}\right|-1\right)$ whenever different individuals $i, j$ belong to the same peer group $P_{m}$, and zero, otherwise.

Notice that one can expand the unidimensional framework described above. Indeed, let $X_{i}$ be a compact subset of the $T$-dimensional Euclidean space $\Re^{T}, T>1$ and define the utility functions as follows:

$$
U(\mathbf{x})=\left\langle\alpha_{i}, x_{i}\right\rangle-\sigma_{i} \frac{\left\|x_{i}\right\|^{2}}{2}+\sum_{j \neq i} \sigma_{i j}\left\langle x_{i}, x_{j}\right\rangle,
$$

where $\|\cdot\|$ and $\langle\cdot, \cdot\rangle$ denote, respectively, the Euclidean norm and the Euclidean scalar product of vectors in $\Re^{T}$. If for every $i \in N$, the set of pure strategies $X_{i}$ is a product set, then the analysis of this game can be undertaken along single dimensions as the utility function is separable across the components. However, if $X_{i}$ is not a product set, then the multidimensional setting cannot be decomposed into several unidimensional components.

\subsection{Neighborhood and Local Interactions}

Glaeser, Sacerdote and Scheinkman (2003) also examine a continuous set of actions, where player $i$ 's utility is given by:

$$
h_{i} x_{i}-E\left\{\sum_{j=1, j \neq i}^{n} J_{i j}\left(x_{i}-x_{j}\right)^{2}\right\}+\epsilon_{i}\left(x_{i}\right), \text { or, } h_{i} x_{i}+2 E\left\{\sum_{j=1, j \neq i}^{n} J_{i j} x_{i} x_{j}\right\}+\epsilon_{i}\left(x_{i}\right) \text {. }
$$

This specification can be decomposed into a private component $h_{i} x_{i}+\epsilon_{i}\left(x_{i}\right)$ and the interaction effect $E\left\{\sum_{j=1, j \neq i}^{N} J_{i j} x_{i} x_{j}\right\}$. The private component can be further 
decomposed into its mean, $h_{i} x_{i}$, and a 0 -mean stochastic deviation $\epsilon_{i}\left(x_{i}\right)$. The terms $J_{i j}$ measure the advantages of conformity: when all $J_{i j}$ are positive, there is an incentive to conform. Our theorem applies when a realization of the random variable $\epsilon_{i}$ is a common knowledge. When the realization is a private information of player $i$, the analysis requires an alternative approach which we will briefly discuss in the next section.

\section{The Binary Setting}

In many social environments, players' strategies are reduced to acceptance or rejection of the action under consideration: ${ }^{7}$ quit smoking (Harris and Lopez-Varcarcel $\underline{(2006)}$ ), become a member of the club (Dixit (2003)), participate in criminal activities (Glaeser, Sacerdote and Scheinkman (1996)), join an industrial alliance (Axelrod et al. (1995)), participate in a riot or a strike (Schelling (1978), Chwe (1999), Granovetter (1978)), choose a side in international conflict (Altfeld and Bueno de Mesquita (1979), Axelrod and Bennett (1993)), purchase a house in a specific residential area (Schelling (1969)), display a national flag on the Independence Day (Chwe (2006)).

\subsection{Critical mass and threshold models of collective action}

A celebrated example of the analysis of the social influence channels in the dichotomic setting has been offered by Shelling (1978). In his threshold model of collective action, the participation of an individual in an action depends on the fraction of the population engaged in the action. This model is a special case of $\left(^{*}\right)$ without the second component, where, moreover, $H(Y, r)=r$ and $H(N, r)=0$ for every positive integer $r$. Indeed, each player $i$ who must chose between participation (Y) and non-participation $(\mathrm{N})$ is represented by a critical mass $t_{i}$ of the number of individuals of players choosing (Y) in order for $i$ to endorse her own participation. That is, for every $i, \alpha_{i}$ is the smallest integer such that $V_{i}(N)-V_{i}(Y) \leq t_{i}$.

In his pioneering paper Granovetter (1978) has pointed out that the influence any given person has on one's decision may also depend on the nature of the pair's relationship. Thus, one needs to reintroduce the second (social interaction) term in $(*)$ to capture heterogeneity of players' dyadic social externalities.

\footnotetext{
${ }^{7}$ See Granovetter (1978) and Schelling (1973) for additional illustrations of this setting.
} 


\subsection{A Theory of International Alliances}

In their strategic analysis of international alignments, ${ }^{8}$ Axelrod and Bennett (1993) (AB - henceforth) developed the so-called landscape theory based on $n$ actors (nations), an $n$-dimensional vector $s$ whose coordinates represent the size (or importance) of the nations, an $n \times n$ matrix $p$, whose entry $p_{i j}$ (positive or negative) represents the propensity of nations $i$ and $j$ to work together. An outcome (configuration) consists of a partition $\pi$ of all nations into two mutually exclusive blocks, $S$ and $T$. AB define the frustration $F_{i}(\pi)$ of country $i \in S$ from $\pi, F_{i}(\pi)=\sum_{j \in T} s_{j} p_{i j}$, and the energy, $E(\pi)$, of the configuration $\pi$ by: $E(\pi)=\sum_{i \in N} s_{i} F_{i}$. AB then examine stable configurations that yield a local minimum of energy over all possible two-bloc alliances. ${ }^{9}$ AB focus on the case where all the entries $p_{i j}$ are symmetric and negative, and define the " distance" between every $i$ and $j$ as $d_{i j}=-p_{i j}>0$. Obviously, the AB model is a special case of our setting, where all players face two choices, $S$ and $T$, in absence of intrinsic preferences for two blocs ${ }^{10}$ and a conformity component, where the dyadic influences are either $-s_{i} s_{j} d_{i j}$ if $i, j$ belong to the same bloc, or 0 , otherwise. ${ }^{11}$

Our model also covers the analysis of standard-setting alliances aiming to develop and sponsor technical standards (Axelrod et al. (1995)). For every firm $i$ the set $N \backslash\{i\}$ is partitioned into two disjoint sets, $C_{i}$ and $D_{i}$, $i$ 's close and distant rivals ${ }^{12}$, respectively. The utility of a firm contemplating to join the alliance $A$ is positively correlated with the size of $A$ but is negatively impacted by allying with its rivals, especially the close ones: $U_{i}=\sum_{j \in A} s_{j} p_{i j}$, where $p_{i j}$ is equal to $1-\alpha$ if $j \in D_{i}$, and to $1-\alpha-\beta$ if $j \in C_{i}$, where $\alpha$ and $\beta$ are two positive parameters. ${ }^{13}$

\footnotetext{
${ }^{8}$ See also the pioneering work of Bueno de Mesquita $(1975,1981)$ on systematic polarity.

${ }^{9} \mathrm{It}$ is interesting to point out that $\mathrm{AB}$ found two stable configurations in Europe, one is the exact partition into the Axis and Allies of World War II, and another that separates the USSR, Yugoslavia and Greece from the rest of Europe.

${ }^{10}$ Galam (1996) removes this restriction.

${ }^{11}$ Note that the AB model is a special case of the multidimensional extension of the linear quadratic-model in subsection 3.1, where the set of players' pure strategies coincides with the vertices of a unit simplex.

${ }^{12}$ This echoes the distinction between strong and weak ties in the analysis of social networks (Granovetter (1973).

${ }^{13}$ Axelrod et al. (1995) test their theory by estimating the choices of nine computer companies to join one of two alliances sponsoring competing UNIX operating system standards in 1988.
} 


\subsection{Local Interaction, Statistical Physics and Ising' Model}

As we indicated in subsection 3.2, the case where the realization of $\epsilon_{i}$ is a private information of player $i$, the analysis of the game requires an alternative approach. Each player $i$ knows the realizations of $\epsilon_{i}$ but has to form beliefs about the strategies of other actors. If the action can only take the values -1 and +1 , we have in equilibrium

$$
\operatorname{Prob}\left(x_{i}=1\right)=F\left(2 h_{i}+4 E\left\{\sum_{j=1, j \neq i}^{N} J_{i j} x_{j}\right\}\right),
$$

where $F$ is the cumulative density function of the random variable $\epsilon_{i}(-1)-\epsilon_{i}(+1)$. There are various specifications of parameters $h_{i}$ and $J_{i j}$. One is the uniform global interaction model considered by Brock and Durlauf (2001) (BD - henceforth) with $h_{i}=h$ and $J_{i j}=\frac{J}{2(N-1)}$, where $h$ and $J$ are two parameters. BD calculate the Nash equilibrium of this mean field model while assuming that each player believes that the expectation of the action of each of his opponents is identical, say $m$. BD derive the equilibrium condition on $m$, and the individual choice probability becomes $\operatorname{Prob}\left(\eta_{i}=1\right)=$ $F(2 h+2 J m)$, where $m=\operatorname{Prob}\left(x_{i}=1\right)-\operatorname{Prob}\left(x_{i}=-1\right)=2 \operatorname{Prob}\left(x_{i}=1\right)-1 . \mathrm{BD}$ derive the equation $m=\tanh \left(\frac{1}{2} g(h+J m)\right)$ where $g(z) \equiv \log F(z)-\log (1-F(z))$. Assuming, in addition, that the random terms are distributed according to the extreme value distribution with parameter $\beta, F(z)=1 /(1+\exp (-\beta z))$, the equation simplifies to $m=\tanh \beta(h+J m)$, which is the well-known Curie-Weiss model of magnetization in statistical physics.

Another important model of social interaction is the uniform local interaction model studied by Blume (1993) and Ellison (1993), where $h_{i}=h$ and $J_{i j}=J$ or 0 , depending upon whether or not $i$ and $j$ are neighbors. ${ }^{14}$ A neighborhood relation can be defined on the undirected graph with players located on the $d$-dimensional integer lattice $Z^{d}$, where neighbors of $i$ are players placed at the minimal distance from $i$, as in the standard Ising's stochastic model.

\section{The Multinomial Framework}

In this section we consider situations where the players face more than two choices. ${ }^{15}$ As an illustration, consider a group of co-workers who make their lunch choices among

\footnotetext{
${ }^{14}$ See the excellent survey of Durlauf (2004) on neighborhood effects.

${ }^{15}$ While we focus on the discrete setting, most of the results can be extended to a continuous case.
} 
$K$ available restaurants, that vary according to the type of cuisine, food quality, price, service, etc. The restaurants offer price discounts for large groups, and the unit lunch price $l_{k}\left(s_{k}\right)$ in restaurant $k$ is decreasing with $s_{k}$, the number of reservations it receives. We assume that the utility of player $i$ choosing restaurant $k$ is $U_{i}(\mathbf{x})=b_{i}^{k}+\sum_{j \in S_{k}} p_{i j}-l_{k}\left(s_{k}\right)$, where the first term refers to the intrinsic benefit of player $i$ from a lunch at restaurant $k$, the second term accounts for the social benefit derived by $i$ from the company of her co-workers $j$ at the lunch table, obtained through dyadic social externalities $p_{i j}$, whereas the last term, previously viewed as conformity effect, accounts for a monetary impact of the restaurant discount. ${ }^{16}$

A specific case of this general framework have been examined by Florian and Galam (2000), who extended the landscape model to include a neutrality as a possible third choice. Brock and Durlauf (2002), Bayer and Timmins (2005) consider a utility specification $U_{i}(\mathbf{x})=b_{i}^{k}+\alpha\left|S^{i}(\mathbf{x})\right|+\epsilon_{i}^{k}$, where $\epsilon_{i}^{k}$ is a random effect on player $i$ from making choice $k$. When the realization of $\epsilon_{i}$ is the private information of player $i$, the equilibrium outcome is described by a probability distribution satisfying some consistency properties.

The existence of Nash equilibria in the case where $U_{i}(\mathbf{x})=V_{i}\left(x_{i}\right)+H\left(x_{i}, \mid S(\mathbf{x} \mid)\right.$, is shown in Konishi, Le Breton and Weber (1997a). In the context of jurisdiction formation, where each jurisdiction selects a local public good and finances it on its own, a Nash configuration in this setting is, in fact, a Tiebout or a sorting equilibrium (Schelling (1978)), where no resident from an existing jurisdiction desires to migrate elsewhere. An equilibrium profile is described by the partition $\pi(\mathbf{x})$ and the actions chosen by coalitions in $\pi(\mathbf{x})$. Here the social interaction game is, in fact, the coalition formation game, where the extent of social influence is limited to members of the same coalition. If functions $V_{i}$ and $H$ are independent of chosen actions, the payoff of a player $i$ is fully determined by the set of players also choosing $i$ 's action, and the coalition formation game becomes hedonic (Banerjee, Konishi and Sömnez (2001), Bogomolnaia and Jackson (2002)). Alternatively, one can generate a hedonic game by ignoring the first and third terms in $\left(^{*}\right)$, and setting $W_{j}^{i}\left(x_{i}, x_{j}\right)$ equal to zero for all $x_{i} \neq x_{j}$, again restricting dyadic social influence to players choosing the same action. ${ }^{17}$

\footnotetext{
${ }^{16}$ One could introduce a congestion effect that may even outweigh price advantages.

${ }^{17}$ In this case our result has been obtained by Bogomolnaia and Jackson (2002).
} 
Finally, the multinomial setting is a natural framework to analyze strategic formation of clusters in social networks, where each player partitions the rest of the society into "friends" and "enemies". The social network is described by a graph $\mathcal{G}$ and the set of vertices $N$, where an (undirected) edge between $i$ and $j$ implies that both $i$ and $j$ consider the other as a friend. Let $U_{i}(\mathbf{x})=\alpha \sum_{j \in S^{i}(\mathbf{x})} d_{i j}+H\left(\left|S^{i}(\mathbf{x})\right|\right)$, where $\alpha$ is a positive parameter and $d_{i j}=1$ if there is an edge between $i$ and $j$, and zero otherwise. In absence of intrinsic preferences over actions, the social heterogeneity is fully described by the graph $\mathcal{G}$, called a sociogram (Moreno (1934)). Our theorem implies the existence of a Nash equilibrium configuration, and, under certain conditions on the parameters, every Nash equilibrium splits the society into cliques of players, each considering the other as a friend. ${ }^{18}$ It is also interesting to point out that by considering the complement of $\tilde{G}$ of $G$ (i.e, players have an edge if they view each other as an enemy), we can utilize the chromatic number of $\tilde{G}$ (Skiena (1990)), which is the smallest number of colors needed to color the vertices of $\tilde{G}$ so that no two adjacent vertices share the same color. Indeed, since adjacent enemies have different colors, the chromatic number provides a lower bound on the number of clusters in a Nash equilibrium partition.

\section{Conclusions}

In this short paper we examine the class of social interaction games where the influence of a peer group is generated through symmetric dyadic interactions between every two members of the group. Given the scope of our note, we sketch a wide range of possible applications, whereas a detailed and more elaborated description of the environments covered by our result, is left for a longer version of the manuscript.

\section{Appendix}

Proof: Consider an $n$-dimensional strategies profile $\mathbf{x}=\left(x_{1}, \ldots, x_{n}\right)$ of players' strategic choices, which generates the partition $\pi(\mathbf{x})=\left(S_{1}, \ldots, S_{K}\right)$ of $N$, where the mem-

\footnotetext{
${ }^{18}$ This notion of a clique (Luce and Perry (1949)) has been extensively examined in sociometry and social network studies. See, e.g., Alba (1973), Seidman and Foster (1978), Borgatti, Everett and Shirey (1990).
} 
bers of each $S_{k}$ choose the same action $x^{k}$. Define the following function ${ }^{19} \Psi$ on $\Lambda=\prod_{i=1}^{n} X_{i}$ :

$$
\begin{array}{r}
\Psi(\mathbf{x})=\sum_{i=1}^{n} V_{i}\left(x_{i}\right)+\frac{1}{2} \sum_{i=1}^{n} \sum_{j=1}^{n} W_{i}^{j}\left(x_{i}, x_{j}\right)+\sum_{k=1}^{K} \sum_{r=1}^{\left|S_{k}\right|} H\left(x^{k}, r\right) \\
=\sum_{i=1}^{n} V_{i}\left(x_{i}\right)+\frac{1}{2} \sum_{i=1}^{n} \sum_{j=1}^{n} W_{i}^{j}\left(x_{i}, x_{j}\right)+\sum_{y \in B(\mathbf{x})} \sum_{r=1}^{|S(\mathbf{x}, y)|} H(y, r),
\end{array}
$$

where $B(\mathbf{x})$ is the range of actions chosen at $\mathbf{x}$, i.e., $B(\mathbf{x})=\left\{y \in X: \exists i\right.$ s. t. $\left.y=x_{i}\right\}$, and $S(\mathbf{x}, y)$ is the cluster of players in $\pi(\mathbf{x})$ who choose $y$. Since, by A1, $V_{i}$ and $W_{i}^{j}$ are upper semi-continuous (usc) for all $i, j \in N$, the first two components of $\Psi$ are usc. We will now show that the third term $\sum_{k=1}^{K} \sum_{r=1}^{\left|S_{k}\right|} H\left(x^{k}, r\right)=\sum_{y \in B(\mathbf{x})} \sum_{r=1}^{|S(\mathbf{x}, y)|} H(y, r)$ is usc as well. Let $y \in B(x)$ and $\left\{\mathbf{x}^{m}\right\}$ be a sequence in $\Lambda$, converging to $\mathbf{x}$. Since for all $j \notin S(\mathbf{x}, y), x_{j} \neq y$, it follows that for $m$ large enough, no action at $\mathbf{x}^{m}$ can be chosen by both a member of $S(\mathbf{x}, y)$ and a player outside of $S(\mathbf{x}, y)$. Thus, again for $m$ large enough, $S(\mathbf{x}, y)$ is the union of sets in $\pi\left(\mathbf{x}^{m}\right)$. Let $B^{m}=$ $\left\{z \in X: \exists i \in S(\mathbf{x}, y)\right.$ s.t. $\left.z=x_{i}^{m}\right\}$. Thus, for $m$ large enough, we obtain by A3:

$$
\sum_{z \in B^{m}} \sum_{r=1}^{\left|S\left(\mathbf{x}^{m}, z\right)\right|} H(z, r) \leq \sum_{r=1}^{|S(\mathbf{x}, y)|} \max _{z \in B^{m}} H(z, r) \equiv \sum_{r=1}^{|S(\mathbf{x}, y)|} H\left(z_{r}^{m}, r\right)
$$

where $H\left(z_{r}^{m}, r\right)=\max _{z \in B^{m}} H(z, r)$ for every $r$. But since $z_{r}^{m}$ converges to $y$ for every $r$ and $H$ is usc with respect to the first argument, $\lim _{n \rightarrow \infty} H\left(z_{r}^{m}, r\right) \leq H(y, r)$. Thus,

$$
\limsup _{m \rightarrow \infty} \sum_{z \in B^{m}} \sum_{r=1}^{\left|S\left(\mathbf{x}^{m}, z\right)\right|} H(z, r) \leq \sum_{r=1}^{|S(\mathbf{x}, y)|} H(y, r) .
$$

Since $X_{i}$ is compact for all $i \in N$, so is $\Lambda$, and the function $\Psi$ being usc, attains its maximum $\mathbf{x}^{*}=\left(x_{1}^{*}, \ldots, x_{n}^{*}\right)$ over $\Lambda$. We claim that $\mathbf{x}^{*}$ is a Nash equilibrium. Assume, in negation, that there exists a player $i \in N$ and a strategy $x_{i} \in X_{i}$ such that $U_{i}(\mathbf{x})<U_{i}(\widetilde{\mathbf{x}})$, where $\widetilde{\mathbf{x}} \equiv\left(x_{i}, x_{-i}^{*}\right)$ :

$V_{i}\left(x_{i}\right)+\sum_{j=1}^{n} W_{i}^{j}\left(x_{i}, x_{j}^{*}\right)+H\left(x_{i},\left|S^{i}(\widetilde{\mathbf{x}})\right|\right)>V_{i}\left(x_{i}^{*}\right)+\sum_{j=1}^{n} W_{i}^{j}\left(x_{i}^{*}, x_{j}^{*}\right)+H\left(x_{i},\left|S^{i}\left(\mathbf{x}^{*}\right)\right|\right)$.

\footnotetext{
${ }^{19}$ Note that in the $\mathrm{AB}$ specification in subsection 4.2, the function $\Phi$ is a weighted sum of players' payoffs, and the potential $\Phi$ coincides with the celebrated fractionalization index ELF (Atlas Narodov Mira, 1964).
} 
Since the number of players who choose $x_{i}$ at $\widetilde{\mathbf{x}}$ exceeds the number of players who chose $x_{i}$ at $\mathrm{x}^{*}$ by one, we have:

$$
\begin{array}{r}
V_{i}\left(x_{i}\right)+\frac{1}{2} \sum_{j=1}^{n} W_{i}^{j}\left(x_{i}, x_{j}^{*}\right)+\frac{1}{2} \sum_{j=1}^{n} W_{j}^{i}\left(x_{j}^{*}, x_{i}\right)+H\left(x_{i},\left|S^{i}\left(\mathbf{x}^{*}\right)\right|+1\right) \\
>V_{i}\left(x_{i}^{*}\right)+\frac{1}{2} \sum_{j=1}^{n} W_{i}^{j}\left(x_{i}^{*}, x_{j}^{*}\right)+\frac{1}{2} \sum_{j=1}^{n} W_{j}^{i}\left(x_{j}^{*}, x_{i}^{*}\right)+H\left(x_{i}^{*},\left|S^{i}\left(\mathbf{x}^{*}\right)\right|\right) .
\end{array}
$$

The last inequality yields

$$
\begin{aligned}
& \Psi(\widetilde{\mathbf{x}})-\Psi\left(\mathbf{x}^{*}\right)=\left[V_{i}\left(x_{i}\right)+\frac{1}{2} \sum_{j=1}^{n} W_{i}^{j}\left(x_{i}, x_{j}^{*}\right)+\frac{1}{2} \sum_{j=1}^{n} W_{j}^{i}\left(x_{j}^{*}, x_{i}\right)+H\left(x_{i}, \mid S^{i}\left(\mathbf{x}^{*} \mid+1\right)\right]\right. \\
& -\left[V_{i}\left(x_{i}^{*}\right)+\frac{1}{2} \sum_{j=1}^{n} W_{i}^{j}\left(x_{i}^{*}, x_{j}^{*}\right)+\frac{1}{2} \sum_{j=1}^{n} W_{j}^{i}\left(x_{j}^{*}, x_{i}^{*}\right)+H\left(x_{i}^{*},\left|S^{i}\left(\mathbf{x}^{*}\right)\right|\right)\right]>0,
\end{aligned}
$$

contradicting the fact that $\mathrm{x}^{*}$ is a maximum of $\Psi$ over $\Lambda$.

\section{References}

Akerlof, G. (1980) "A theory of social custom, of which unemployment may be one consequence", Quarterly Journal of Economics 94, 749-775.

Akerlof, G. (1997) "Social distance and social decisions", Econometrica 65, 10051027.

Alba, R. (1973) "A graph-theoretic definition of a sociometric clique", Journal of Mathematical Sociology 3, 113-126.

Altfeld, M. and B. Bueno de Mesquita (1979) "Choosing sides in war", International Studies Quarterly 23, 87-112.

Atlas Narodov Mira (1964). Moscow: Miklukho-Maklai Ethnological Institute at the Department of Geodesy and Cartography of the State Geological Committee of the Soviet Union.

Axelrod, R. and D. Bennett (1993) "A landscape theory of aggregation", British Journal of Political Science 23, 211-233.

Axelrod, R., Mitchell, W., Thomas, R., Bennett, D. and E. Bruderer (1995) "Coalition formation in standard-setting alliances", Management Science 41, 14931508. 
Ballester, C., Calvó-Armengol, A. and Y. Zenou (2006) "Who's who in networks. Wanted: the key player", Econometrica 74, 1403-1417.

Banerjee, S., Konishi, H. and T. Sömnez (2001) "Core in a simple coalition formation game", Social Choice and Welfare 18, 135-153.

Bayer, P. and C. Timmins (2005) "On the equilibrium properties of locational sorting models", Journal of Urban Economics 57, 462-477.

Becker, G. (1974) "A theory of social interactions", Journal of Political Economy 82, 1063-1093.

Bernheim, B.D. (1994), "A theory of conformity", Journal of Political Economy 102, 841-877.

Blume, L. (1993) "The statistical mechanics of strategic interaction", Games and Economic Behavior 5, 387-424.

Blume, L. and S. Durlauf (2001) "The interactions-based approach to socioeconomic behavior", in Social Dynamics, S. Durlauf and P. Young, eds., MIT Press, Cambridge.

Blume, L. and S. Durlauf (2003) "Equilibrium concepts for social interaction models", International Game Theory Review 5, 193-209.

Bogomolnaia, A. and M. Jackson (2002) "The stability of hedonic coalition structures" Games and Economic Behavior 38, 201-230.

Borgatti, S., Everett, M. and P. Shirey (1990) "LS sets, lambda sets, and other cohesive subsets", Social Networks 12, 337-358.

Brock, W. and S. Durlauf (2001) "Discrete choice with social interactions", Review of Economic Studies 68, 235-260.

Brock, W. and S. Durlauf (2002) "A multinomial choice model with neighborhood effects", American Economic Review 92, 298-303.

Bueno de Mesquita, B. (1975) "Measuring systemic polarity", Journal of Conflict Resolution 19, 187-216.

Bueno de Mesquita, B. (1981) The War Trap, Yale University Press, New Haven.

Corbo, J., Calvó-Armengol, A. and D. Parkes (2007) "The importance of network topology in local contribution games" in The Third International Workshop on Internet and Network Economics, X. Tie and F. Chung Graham, eds., Springer Verlag.

Chwe, M.S. (1999) "Structure and strategy in collective action", American Journal 
of Sociology 105, 128-156.

Chwe, M.S. (2006) "Incentive compatibility implies signed covariance", UCLA, Mimeo.

Dixit, A. (2003) "Clubs with entrapment", American Economic Review 93, 18241829.

Durlauf, S. (1999) "How can statistical mechanics contribute to social science?", Proceedings of the National Academy of Sciences 96, 10582-10584.

Durlauf, S. (2004) "Neighborhood Effects", in Handbook of Regional and Urban Economics, Vol. 4, J.V. Henderson and J.F. Thisse, eds., North-Holland, Amsterdam.

Ellison, G. (1993) "Learning, local interaction and coordination", Econometrica 61, 1047-1072.

Fafchamps, M. and F. Gubert (2007) "The formation of risk sharing networks", Journal of Development Economics 83, 326-350.

Florian, R. and S. Galam (2000) "Optimizing Conflicts in the Formation of Strategic Alliances", European Physical Journal 16, 189-194.

Galam, S. (1996) "Fragmentation versus stability in bimodal coalitions", Physica 230, 174-188.

Galeotti, A., Goyal, S., Jackson, M. Vega-Redondo, F. and L. Yariv (2006) "Network games", California Institute of Technology, mimeo.

Glaeser, E., Sacerdote, B. and J. Scheinkman (1996) "Crime and social interactions", Quarterly Journal of Economics 111, 507-548.

Glaeser, E., Sacerdote, B. and J. Scheinkman (2003) "The social multiplier", Journal of the European Economic Association 1, 345-353.

Glaeser, E. and J. Scheinkman (2003) "Non-market interventions", in Advances in Economics and Econometrics: Theory and Applications, Eight World Congress, Vol. 1, M. Dewatripont, L.P. Hansen and S. Turnovsky, eds., Cambridge University Press, 339-369.

Granovetter, M. (1973) "The strength of weak ties", American Journal of Sociology $78,1360-1380$.

Granovetter, M. (1978) "Threshold models of collective action", American Journal of Sociology 83, 1420-1443.

Harris, J. and B. Lopez-Varcarcel (2006) "Asymmetric social interaction in economics: cigarette smoking among young people in the U.S. 1992-1993”, NBER Work- 
ing Paper 10409.

Jackson, M (2008) "Average distance, diameter and clustering in social networks with homophily", Stanford University, mimeo.

Jones, S. (1984), The economics of conformism, Blackwell, Oxford, U.K.

Konishi, H., Le Breton, M. and S. Weber (1997a) "Pure strategy Nash equilibria in a group formation game with positive externalities", Games and Economic Behavior 21, 161-182.

Konishi, H., Le Breton, M. and S. Weber (1997b) "Equilibria in a model with partial rivalry", Journal of Economic Theory 72, 225-237.

Kukushkin, N. (2007) "Congestion games revisited", International Journal of Game Theory 36, 57-83.

Luce R. and A. Perry (1949) "A method of matrix analysis of group structure", Psychometrika 14, 94-116.

Manski, C. (2000) "Economic analysis of social interactions", Journal of Economic Perspectives 14, 115-136.

Milchtaich, I. (1996) "Congestion games with player-specific payoff function", Games and Economic Behavior 13, 111-124.

Monderer, D. and L. Shapley (1996) "Potential games", Games and Economic Behavior 14, 124-143.

Moreno, J. (1934) "Who shall survive? A new approach to the problem of human interrelations", New York: Beacon House.

Rosenthal, R. (1973) "A class of games possessing a pure-strategy Nash equilibrium", International Journal of Game Theory 2, 65-67.

Schelling, T. (1969) "Models of segregation", American Economic Review, Papers and Proceedings 59, 488-493.

Schelling, T. (1973) "Hockey helmets, concealed weapons, and daylight savings: a study of binary choices with externalities", Journal of Conflict Resolution 17, 381-428.

Schelling, T. (1978) Micromotives and Macrobehavior, Norton, New-York.

Seidman, S. and B. Foster (1978) "A graph-theoretic generalization of the clique concept", Journal of Mathematical Sociology 6, 139-154.

Skiena, S. (1990) "Line graph" in Implementing Discrete Mathematics: Combinatorics and Graph Theory with Mathematica, Addison-Valley, Reading, MA. 